\title{
Supporting Information: Anisotropic Strain in Rare-Earth Substituted Ceria Thin Films Probed by Polarized Raman Spectroscopy and First-Principles Calculations
}

Eva Sediva, ${ }^{1}$ Dmytro Bohdanov, ${ }^{1}$ George F. Harrington, ${ }^{2,3}$ Iegor Rafalovskyi, ${ }^{1}$ Jan Drahokoupil, ${ }^{1}$ Fedir Borodavka, ${ }^{l}$ Pavel Marton, ${ }^{l}$ Jiri Hlinka ${ }^{l}$

1 Institute of Physics of the Czech Academy of Sciences, Na Slovance 1999, Prague 18221, Czech Republic

2 Center of Coevolutionary Research for Sustainable Communities ( ${ }^{2}$ RSC), Kyushu University, 744 Motooka, Nishi-ku, Fukuoka 819-0395, Japan

3 Department of Materials Science and Engineering, Massachusetts Institute of Technology, 77 Massachusetts Ave., Cambridge, MA, 02139, USA 


\section{$1 \quad$ X-Ray Diffraction}

Figure $\mathrm{S} 1 \mathrm{a}$ and $\mathrm{b}$ shows example scans for the $\mathrm{Yb}: \mathrm{CeO}_{2}$ sample around the 006 and 404 film reflections respectively. For the purposes of calculating the lattice parameters, only the central part of the film reflections without the substrate were precisely mapped. Particular $2 \theta$ positions were determined by the maximal intensity. $2 \theta-\omega$ scans with highest intensity are plotted in Figure S1 c and d. Lattice constants are summarized in Table S1.
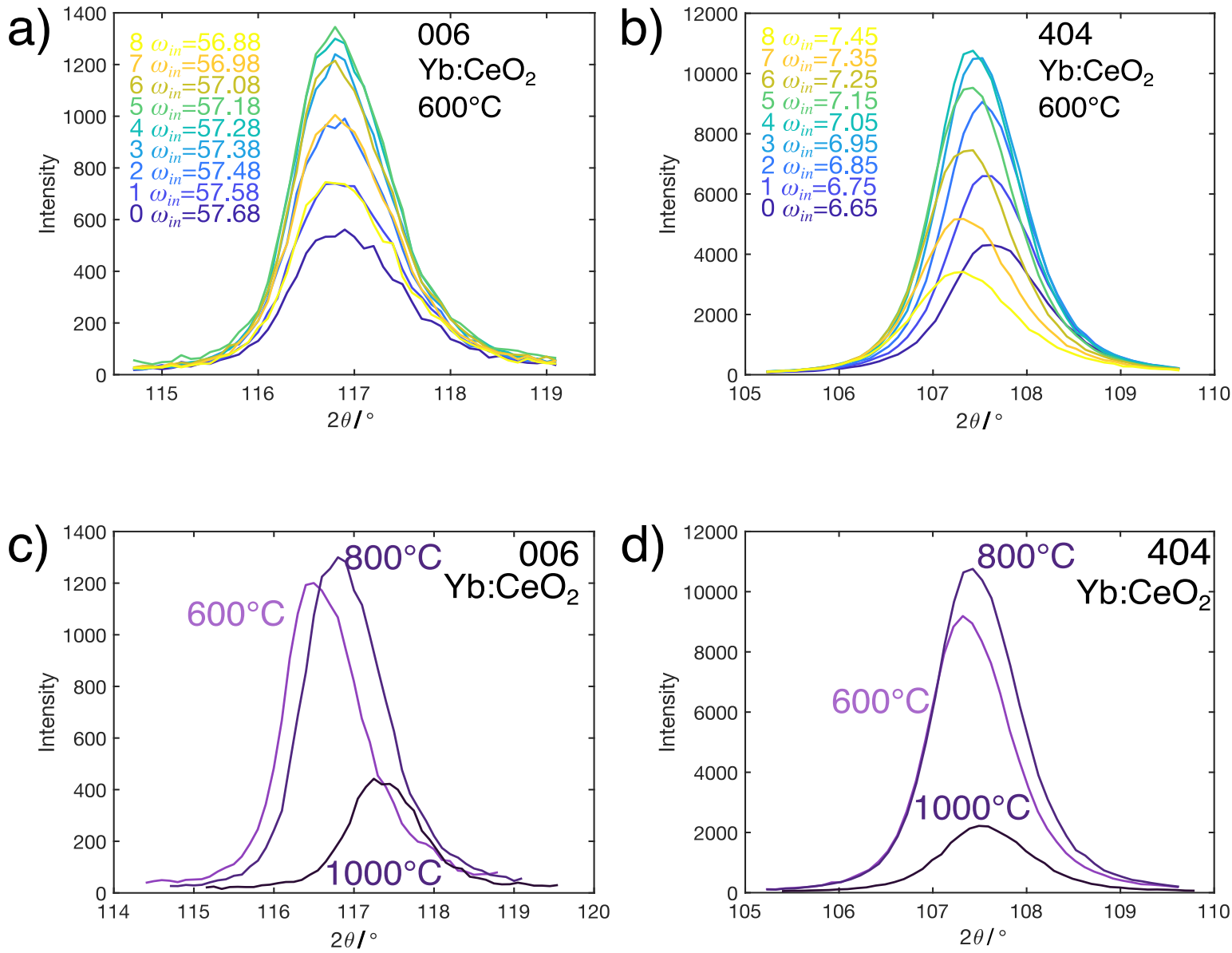

Figure S1: Example of the X-Ray diffraction scans for the $\mathrm{Yb}: \mathrm{CeO}_{2}$ thin films. $92 \theta-\omega$ scans around the 006 a) and 404 b) film reflections. The scan at the intensity maximum for each strain level around the 006 c) and 404 d) film reflections. 
Table S1: Summary of in- and out-of-plane lattice parameters measured by X-Ray diffraction for all thin film samples.

\begin{tabular}{|c|c|c|c|c|}
\hline Annealing & & & & Volume \\
\hline Temp. & $\begin{array}{l}\text { Gd: } \mathrm{CeO}_{2} \\
\text { a (404) [Å] }\end{array}$ & c (006) [Å] & c/a-1 & $\left.\AA^{3}\right]$ \\
\hline $600^{\circ} \mathrm{C}$ & 5.399 & 5.430 & 0.0056942 & 158.3165 \\
\hline $800{ }^{\circ} \mathrm{C}$ & 5.402 & 5.431 & 0.005427 & 158.4596 \\
\hline $1000^{\circ} \mathrm{C}$ & 5.398 & 5.426 & 0.005162 & 158.1012 \\
\hline & $\begin{array}{r}\text { Yb: } \mathrm{CeO}_{2} \\
\text { a (404) }\end{array}$ & c (006) & c/a-1 & \\
\hline $600^{\circ} \mathrm{C}$ & 5.387 & 5.435 & 0.009067 & 157.7130 \\
\hline $800^{\circ} \mathrm{C}$ & 5.394 & 5.428 & 0.006394 & 157.9177 \\
\hline $1000^{\circ} \mathrm{C}$ & 5.397 & 5.414 & 0.003085 & 157.6790 \\
\hline & $\begin{array}{c}\mathrm{La}: \mathrm{CeO}_{2} \\
\text { a }(404)\end{array}$ & c (006) & c/a-1 & \\
\hline $600{ }^{\circ} \mathrm{C}$ & 5.404 & 5.451 & 0.008761 & 159.1906 \\
\hline $800^{\circ} \mathrm{C}$ & 5.404 & 5.447 & 0.007942 & 159.0588 \\
\hline $1000^{\circ} \mathrm{C}$ & 5.407 & 5.440 & 0.006104 & 159.0563 \\
\hline
\end{tabular}

2 Ab initio calculations of the Raman mode frequencies

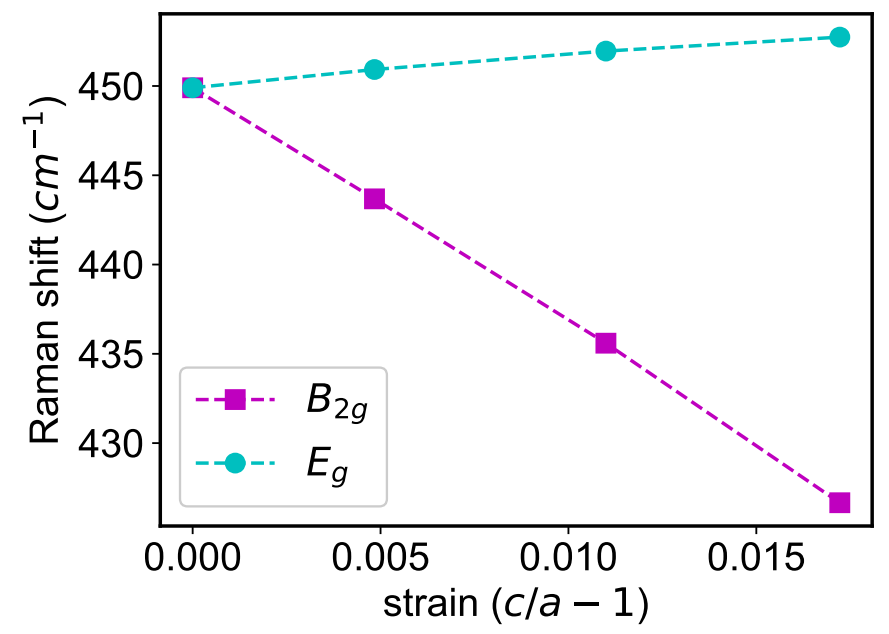

Figure S2: Positions of the $B_{2 g}$ and $E_{g}$ modes as a function of strain as calculated by ab initio calculations. 


\section{Example Raman spectra and fits}

a) thin films, $z(x x) \bar{z}$

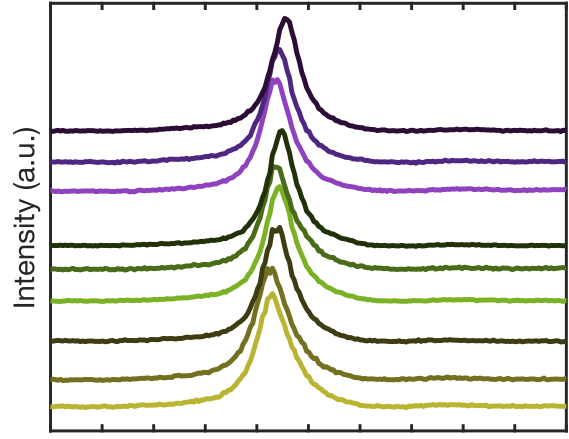

350375400425450475500525550575600

Raman shift $\left(\mathrm{cm}^{-1}\right)$

b)

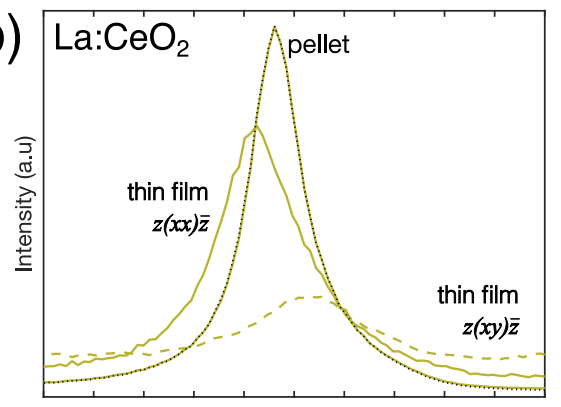

$\begin{array}{lllllllllll}415 & 425 & 435 & 445 & 455 & 465 & 475 & 485 & 495 & 505 & 515\end{array}$ Raman shift $\left(\mathrm{cm}^{-1}\right)$

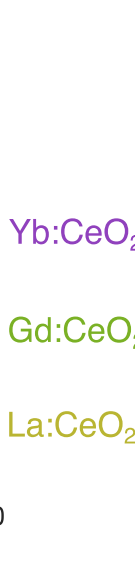

thin films, $z(x y) \bar{z}$

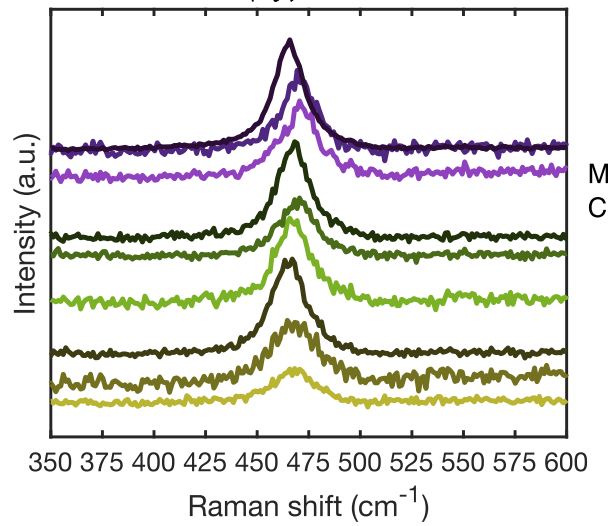

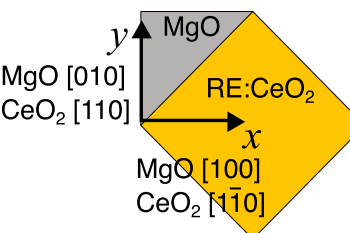

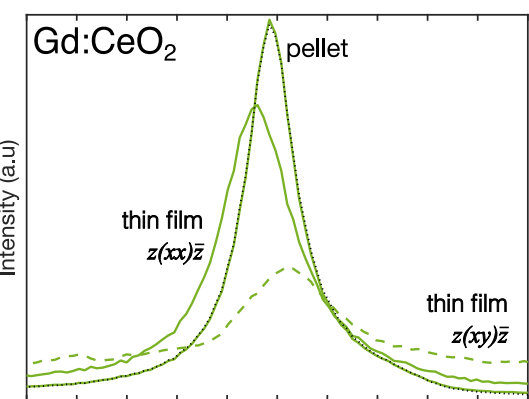

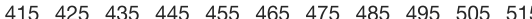
Raman shift $\left(\mathrm{cm}^{-1}\right)$

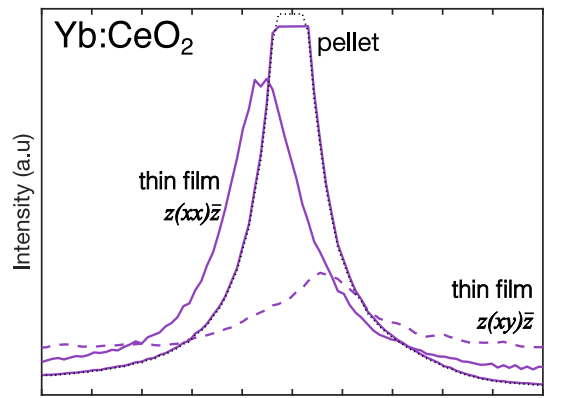

$\begin{array}{llllllllllll}415 & 425 & 435 & 445 & 455 & 465 & 475 & 485 & 495 & 505 & 515\end{array}$ Raman shift $\left(\mathrm{cm}^{-1}\right)$

Figure S3 a. Raman spectra for all thin films with different dopants and strains in the backscattering geometry and the $x x$, and $x y$ polarization configurations. $b$. Comparison of the $x x, x y$ and pellet spectra for three different samples. (The flat peak of the pellet response in $\mathrm{Yb}: \mathrm{CeO}_{2}$ signifies saturation of the detector.)

The thin film spectra in Figure S2 were integrated for $20 \mathrm{~s}$ and averaged over 8 accumulations. 

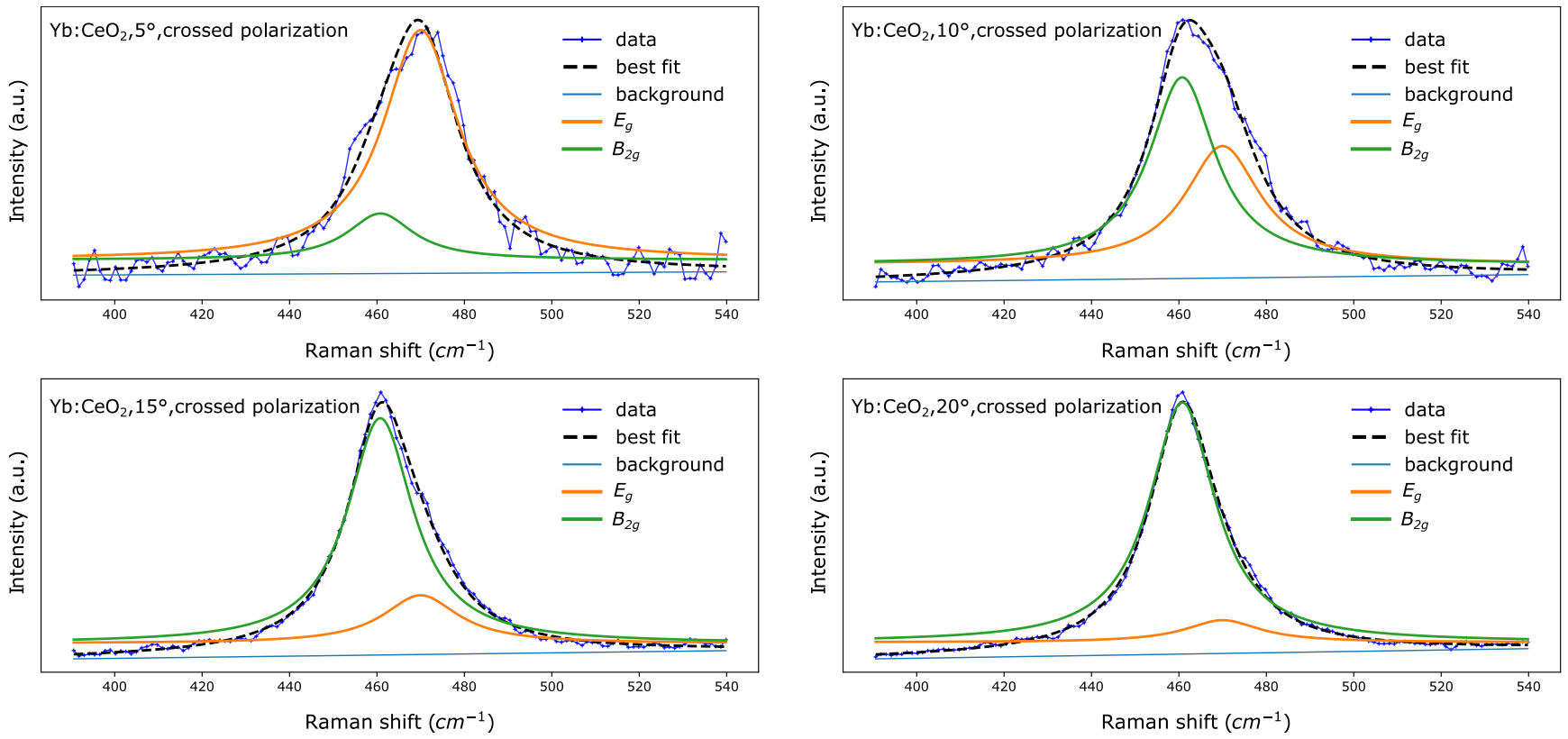

Figure S4 Example fits of the $\mathrm{Yb}: \mathrm{CeO}_{2}$ thin film measured at arbitrary angles. The positions and full width at half maximum of the $B_{2 g}$ and $E_{g}$ modes have been fixed by the $z(x x) \bar{z}$ and $z(x y) \bar{z}$ measurements respectively.

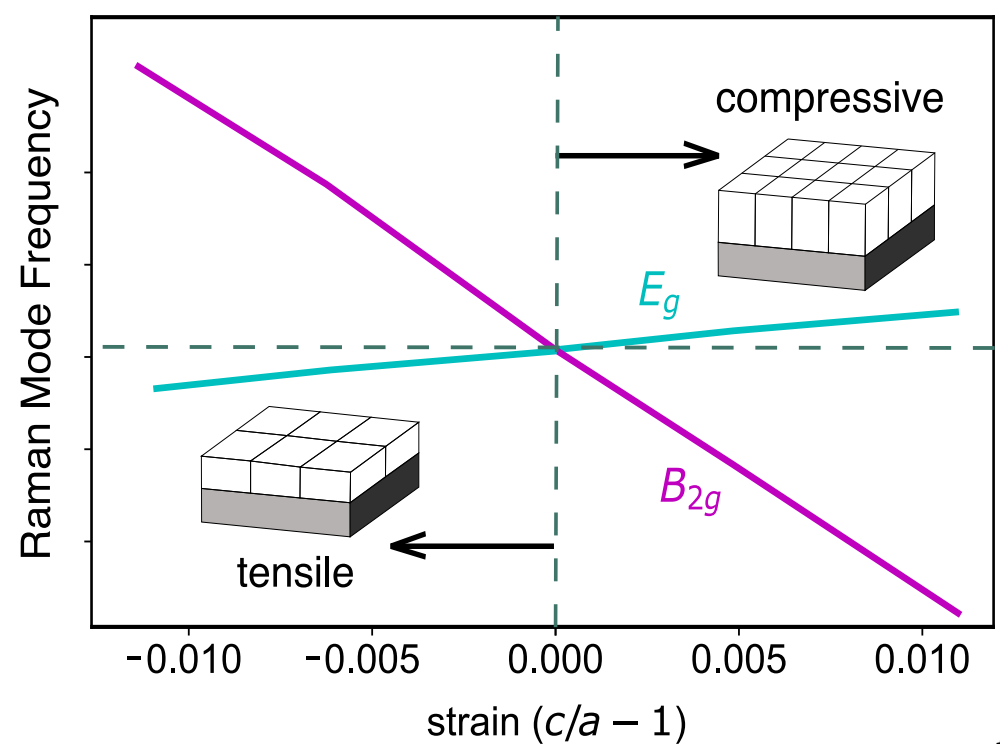

and $B_{2 g}$ modes for both tensile and compressive strain.

Figure S5: Sketch of the behavior of the $E_{g}$ 